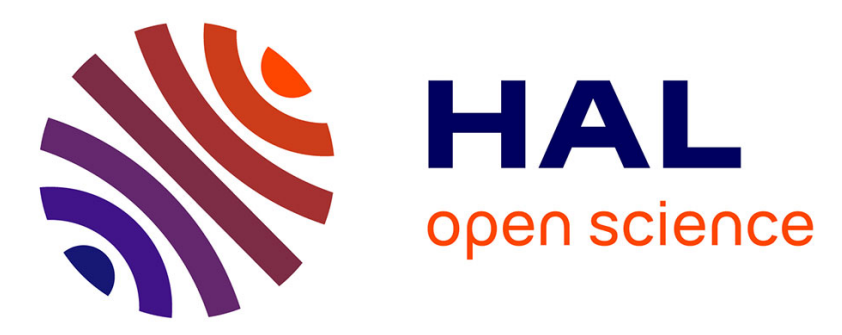

\title{
Action-Self Quenching: Dimer-Induced Fluorescence Quenching of Chromophores as a Probe for Biomolecular Structure
}

Steven Daly, Chang Min Choi, Fabien Chirot, Luke Macaleese, Rodolphe Antoine, Philippe Dugourd

\section{To cite this version:}

Steven Daly, Chang Min Choi, Fabien Chirot, Luke Macaleese, Rodolphe Antoine, et al.. Action-Self Quenching: Dimer-Induced Fluorescence Quenching of Chromophores as a Probe for Biomolecular Structure. Analytical Chemistry, 2017, 89 (8), pp.4604-4610. 10.1021/acs.analchem.7b00152 . hal01539953

\section{HAL Id: hal-01539953 https://hal.science/hal-01539953}

Submitted on 28 Oct 2020

HAL is a multi-disciplinary open access archive for the deposit and dissemination of scientific research documents, whether they are published or not. The documents may come from teaching and research institutions in France or abroad, or from public or private research centers.
L'archive ouverte pluridisciplinaire HAL, est destinée au dépôt et à la diffusion de documents scientifiques de niveau recherche, publiés ou non, émanant des établissements d'enseignement et de recherche français ou étrangers, des laboratoires publics ou privés. 


\title{
Action-Self Quenching: Dimer-induced Fluorescence Quenching of Chromophores as a Probe of Biomolecules.
}

\author{
Steven Daly ${ }^{\mathrm{a}}$, Chang Min Choi ${ }^{\mathrm{a}:}$, Fabien Chirot $^{\mathrm{b}}$, Luke MacAleese $^{\mathrm{a}}$, Rodolphe Antoine ${ }^{\mathrm{a}}$, Philippe Du- \\ $\operatorname{gourd}^{\mathrm{a}^{*}}$.

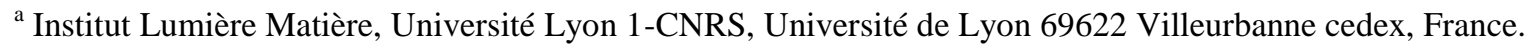 \\ ${ }^{\mathrm{b}}$ Institut des Sciences Analytiques, Université Lyon 1 - CNRS, Université de Lyon, 69622 Villeurbanne Cedex, France.
}

\begin{abstract}
A detailed understanding of the interplay between structure and interactions of biomolecular systems is of high importance in order to map biomolecule function and to develop new targets for treatment of diseases. In parallel to in vivo and in vitro studies, methods for probing biomolecular structure in vacuo have also been developed; for example native mass spectrometry, ion mobility mass spectrometry, and FRET techniques. Here, we propose a novel technique for probing biomolecular structure based on the changes in photophysics of a rhodamine-based chromophore upon dimer formation, which we call action selfquenching. Comparison of solution and gas phase measurements on a doubly tagged tripeptide shows that fluorescence quenching in the chromophore dimer is accompanied by an increase in photofragmentation yield. Two test systems of different size - the 1228 fragment of Amyloid beta, and ubiquitin - are then used to probe the sensitivity and applicability of action self-quenching. A particularly important observation is that action self-quenching is capable of probing very small changes in conformation as it shows an on-off response, making it complementary to FRET based techniques, which are insensitive at very short chromophore separations.
\end{abstract}

\section{Introduction.}

A detailed understanding of the interplay between structure and interactions of biomolecular systems is of high importance in order to map biomolecule function and to develop new targets for treatment of diseases. Amongst the techniques used to probe biomolecules, those based around fluorescence are perhaps the most commonly used. ${ }^{1-7}$ In particular, Förster Resonance Energy Transfer (FRET) has become a ubiquitous technique for probing changes in protein structure and association. In parallel to in vivo and in vitro studies, methods for probing biomolecular structure in vacuo have also been developed. Although mass spectrometry has been used as a tool in proteomics for many years, the recent advent of native mass spectrometry has allowed gas phase studies to progress from simple sequencing to stoichiometric and structural measurements. ${ }^{8-10}$ Strategies combining native mass spectrometry and ion-mobility mass spectrometry (IM-MS) are now well attested as sensitive probes to the gas-phase conformations of biomolecular systems. ${ }^{11}$ Recently, FRET has also been transposed to the gas-phase, being evidenced both by fluorescence detection, and by detection of specific photofragmentation (actionFRET). ${ }^{12-21}$ Action-FRET has been used to explore the conformational landscape of different alloforms of a fragment of the amyloid beta peptide, as well as dimer formation. ${ }^{17,22}$ More recently, FRET has been applied to full-sized proteins for the first time in the gas-phase, providing evidence that the technique will be a useful addition to the gas-phase structural biology toolbox. ${ }^{15,18,21}$
One of the biggest advantages of performing FRET measurements is that they have the potential to provide a direct link between conformational ensembles in the solution and gas phase. It has long since been demonstrated that soft ionization mechanisms such as electrospray ionization (ESI) can preserve solution phase conformational ensembles into the gas phase. The nature of this link and the evolution of the conformational ensemble upon transposition to the gas-phase has been more difficult to pin down and is an active area of current research. ${ }^{9,23-27}$ The processes that occur during FRET are essentially identical in solution and gas phase (the differences being changes in optical properties in different media which may be accounted for computationally). The measurement of FRET efficiencies in the gas- and solution phases may thus be directly compared. ${ }^{28}$ This unique correspondence has the potential to answer some of the most fundamental questions remaining in native mass spectrometry from the structural biology perspective.

FRET, however, does have some limitations. Firstly, the prerequisite of two chromophores (donor and acceptor) to be tagged onto the target protein (or proteins) means that preferably two specific tagging locations must be present. If the same tagging chemistry is used for both chromophores, there will be a lack of specificity which leads to multiple species being present in solution (donor-donor, donor-acceptor, acceptordonor, and acceptor-acceptor). Although this is not a problem in the gas-phase, where differences in the molecular weight allows easily isolation of a specific species (except for the donor $\leftrightarrow$ acceptor case, where the two products are isobaric), extra purification steps are necessary for solution-phase stud- 
ies to achieve the same purity, and the concentration of FRETactive protein is reduced. Alternatively it is possible to use either protecting groups, unnatural amino acids using stopcodon mutations, or tagging during the synthesis step directly to avoid such multiplicity, but this can greatly complicate an experimental methodology. ${ }^{29-31}$ Second, although FRET is very sensitive in the $1-10 \mathrm{~nm}$ range (depending upon the chromophore pair chosen), it breaks down at very short distances, where the point-dipole approximation fails, and where it is not possible to consider the chromophores as independent systems. $^{32}$ This precludes the use of FRET to study, for example, small conformational changes induced by substrate binding to a protein.

Certain fluorescent probes used in FRET experiments have a further property which is useful in overcoming both aforementioned limitations, namely self-quenching. It is well known that rhodamine derivatives undergo fluorescence quenching at high concentrations. This has been attributed to energy hopping to dimeric species, which act as quenching centers, reducing the fluorescence lifetime from a few nanoseconds to as low as 1 picosecond. ${ }^{33}$ The mechanism of fluorescence quenching in rhodamine dimers has been investigated by Setiawan et al., who showed that internal conversion following photoexcitation to an optically bright state to a lower lying optically dark state is responsible. ${ }^{34}$ Interestingly, it was also found that the relative orientation of the chromophores has a strong influence on the relative ordering of the dark and bright states. ${ }^{34}$ Furthermore, a single-molecule study by Hernando et al. on tetramethylrhodamine dimers have shown that there exist strongly and weakly bound dimers, differing by the separation of the chromophores. ${ }^{35}$ These results showed that it was only strongly interacting chromophores with separations less than $1 \mathrm{~nm}$ which undergo self-quenching.

The short-distance dependence of such self-quenching measurements has been used to study, for example, the aggregation of amyloid beta fibrils, and the folding kinetics of leucine zipper domains. ${ }^{36,37}$ Chromophore dimer formation as a mechanism for probing protein conformational dynamics is particularly attractive since it is extremely sensitive to small changes in the separation. Indeed, Zhou et al. showed that a sub-nanometer resolution is possible, observing a 20 fold increase in fluorescence yield following ADP binding to the ParM protein which corresponds to an increase of only $0.5 \mathrm{~nm}$ in tagging site separation. ${ }^{38}$ This makes dimer-induced selfquenching a suitable complement to FRET experiments, which are more sensitive to larger conformational changes.

In this paper, the feasibility of using dimer-induced selfquenching of fluorescence in Atto 520 - a rhodamine analogue closely related to rhodamine $6 \mathrm{G}-$ as a probe of structural change in the gas phase was examined using a variety of systems of different size.

\section{Methodology}

\section{Sample preparation}

Atto 520 maleimide (A520, Figure S1) (AttoTec GmbH, Germany) was dissolved in DMSO to a final concentration of $20 \mathrm{mM}$. This was subsequently diluted in a $1: 1 \mathrm{v} / \mathrm{v}$ mixture of either $\mathrm{H}_{2} \mathrm{O}: \mathrm{CH}_{3} \mathrm{OH}$ or $\mathrm{H}_{2} \mathrm{O}: \mathrm{CH}_{3} \mathrm{CN}$ to a concentration of 100 $\mu \mathrm{M}$ as stock solution for the reactions. $\mathrm{H}-\mathrm{CAC}-\mathrm{OH}$ (genecust, Luxembourg) was dissolved in $1: 1 \mathrm{v} / \mathrm{v}_{2} \mathrm{O}: \mathrm{CH}_{3} \mathrm{OH}$ to a con- centration of $100 \mu \mathrm{M}$. The 12-28 fragment of wild-type amyloid beta with terminal cysteine residues (aceCVHHQKLVFFAENVGSNKC-NH ${ }_{2}$, Genecust, Luxembourg) was dissolved in $1: 1 \mathrm{v} / \mathrm{v} \mathrm{H}_{2} \mathrm{O}: \mathrm{CH}_{3} \mathrm{CN}$ to a final concentration of $100 \mu \mathrm{M}$. A double cysteine mutant (G35C L73C, Genescript, USA) was purchased and purified as described in detail elsewhere. $^{18}$

Tagging of the peptides was performed by dilution to the desired final concentration of the peptide $(10 \mu \mathrm{M}$ for gasphase action spectroscopy measurements, $1 \mu \mathrm{M}$ for reaction kinetic measurements in solution and gas phase), and addition of chromophore in a 2:1 molar ratio so that tagging could be performed with close to $100 \%$ yields. All reaction mixtures were tested via ESI-MS to ensure the tagging reaction had run to completion.

\section{Reaction Monitoring}

Fluorescence intensity measurements were performed in a Fluoromax-4 spectrophotometer (Horiba). Final peptide concentrations of $1 \mu \mathrm{M}$ were used, corresponding to $2 \mu \mathrm{M}$ chromophore concentration. Fluorescence excitation was performed at $520 \mathrm{~nm}$ with a $1 \mathrm{~nm}$ bandwidth and fluorescence was monitored every 60 seconds at $543 \mathrm{~nm}$ (fluorescence maximum, Figure S2) with $1 \mathrm{~nm}$ bandwidth. To perform the measurement, $3 \mathrm{ml}$ of $1 \mu \mathrm{M}$ solution of CAC in $\mathrm{H}_{2} \mathrm{O}: \mathrm{CH}_{3} \mathrm{OH}$ was added to the cuvette within the spectrometer. Immediately prior to starting the acquisition, a two-fold molar excess of A520 was added to the solution in the cuvette in order to minimize time lag between addition of the chromophore and commencement of the measurement.

For gas phase measurements, an identical solution was prepared just prior to starting a measurement and was injected directly into the ESI source. The relative intensity of the $1+$ and $2+$ charge states of singly tagged CAC, and the $2+$ and $3+$ of doubly tagged CAC (each chromophore carries a permanent positive charge, and a single protonation site - the $\mathrm{N}$-terminus - on the peptide) was monitored as a function of time. The total mass spectrum was recorded continuously until the sample was fully injected, approximately 60 minutes after injection was commenced.

To generate a reconstructed fluorescence intensity profile, the maximum and final fluorescence intensity measured in solution were convoluted with the relative intensities of the singly and doubly tagged peptide respectively; $I_{\text {reconstruct }}=$ $\left(F I_{\text {max }} * I_{S G}\right)+\left(F I_{\text {end }} * I_{D G}\right)$ where $F I_{\text {max }}$ and $F I_{\text {end }}$ are the maximum and final fluorescence intensities, and $I_{S G}$ and $I_{D G}$ are the relative intensity of singly and double tagged peptide respectively.

\section{Optical Spectroscopy.}

The experimental setup used for optical action-spectroscopy measurements has been described in detail elsewhere. ${ }^{39}$ Briefly, a linear quadrupole ion trap mass spectrometer (LTQ Velos, Thermo Fisher Scientific, San Jose, CA) was used to generate, mass select, and trap ions in a first, high pressure trap for a controlled period during which they may be activated by laser irradiation (laser induced dissociated, LID). After irradiation ions are transmitted to a second, lower pressure ion trap, to be mass analyzed. A fused silica window (3 mm thick, 1 in. diameter) is positioned at the back end of the instrument, and 1-2 mm diameter circular openings in the trapping elec- 
trodes allow coupling of light source and trapped ions. In order to optimize laser transmission, the central hole of the electrode closest to the fused silica window was enlarged to 5 $\mathrm{mm}$ in diameter.

The light source used was a Horizon OPO pumped by the third harmonic of a Surelite II Nd:YAG laser (Continuum, Santa Clara , CA). A repetition rate of $10 \mathrm{~Hz}$ and pulse widths of $5 \mathrm{~ns}$ were used. The beam was focused into the first ion trap using a $1000 \mathrm{~mm}$ focal length convergent lens. A mechanical shutter, synchronized with the mass spectrometer, was used to stop the beam at all times except the "ion activation window", that is, the time after ion accumulation and before mass analysis. A single laser pulse was used for the irradiation of the trapped ions, and the normalized collision energy is kept at zero during irradiation.

To record action spectra, the laser wavelength was scanned between 530 and $420 \mathrm{~nm}$, in $0.1 \mathrm{~nm}$ steps and with a $1 \mathrm{~s}$ dwell time at each wavelength, resulting in a total acquisition time of approximately 20 minutes. Mass spectra were recorded every $9 \mu$ scan, which corresponded to approximately one mass spectrum per second. Laser power was recorded by performing a second scan under identical conditions immediately following acquisition of the action spectrum. To analyse the data, each mass spectrum was assigned a wavelength value given by $\lambda_{\text {start }}-\left((i-1) *\left(\frac{\Delta \lambda}{n}\right)\right)$ where $\lambda_{\text {start }}=530 \mathrm{~nm}$ is the starting wavelength, $i$ the mass spectrum number, $\Delta \lambda=110 \mathrm{~nm}$ is the total wavelength change, and $n$ the total number of mass spectra acquired during the acquisition. The same procedure was used to provide a wavelength range for the laser power $(P)$, and a linear interpolation performed to give values of the laser power at the wavelengths assigned to each mass spectrum. The fragmentation yield was then defined as $-\log \left(I_{\text {parent }} / I_{\text {total }}\right) /(\lambda * P)$ where $I_{\text {parent }}$ and $I_{\text {total }}$ are the parent and total ion signal respectively at each point in the acquisition. For the doubly-tagged amyloid beta peptides, specific fragmentation yields are used and defined as $I_{S F Y} / I_{\text {total }} /(\lambda * P)$ where $I_{S F}$ is the intensity of the specific fragments considered (see details below).

For ubiquitin, where the photofragmentation is much lower, a background subtraction was performed on the specific fragmentation yield. To do this, a peak-free region of the mass spectrum was chosen and the intensity summed over a range of identical size as used to determine the specific fragmentation yield. The background corrected specific fragment intensiy was then calculated as $I_{C S F Y}=I_{S F Y}-I_{b g}$, and the specific fragmentation yield determined as above to be $I_{C S F Y} / I_{\text {total }}$.

\section{Ion Mobility Mass Spectrometry.}

Ion mobility measurements were performed on a tandem drift tube mass spectrometer described in detail elsewhere. ${ }^{40,41}$ Here ions were analyzed using 4 Torr helium as a buffer gas at room temperature and utilizing a drift voltage of $400 \mathrm{~V}$.

\section{Results and Discussion}

In solution, self-quenching can be relatively easily evidenced by changes in either the fluorescence quantum yield or the fluorescence lifetime. Direct gas-phase measurements of these properties are much more challenging, and beyond the capabilities of the experimental setup used here. It is therefore worthwhile taking some time to examine and compare solution

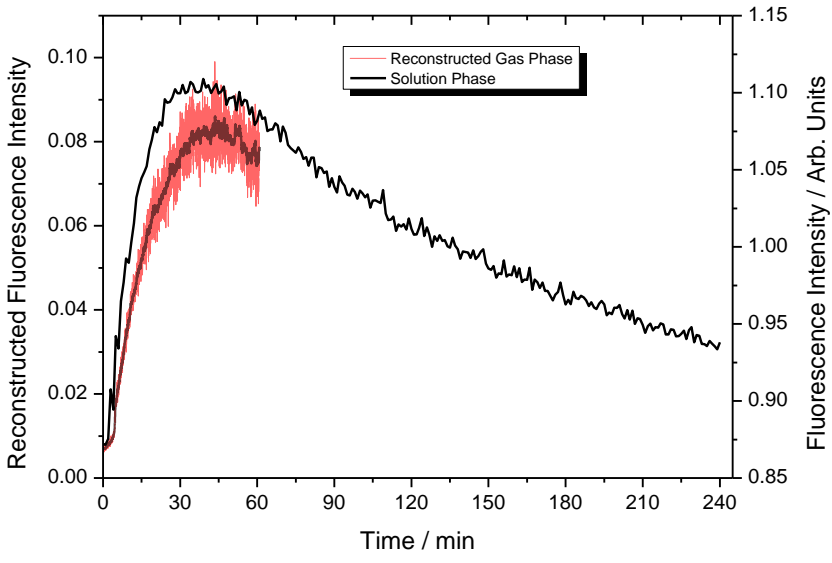

Figure 1. Fluorescence intensity (solid black line) at $543 \mathrm{~nm}$ of a 2:1 ratio of Atto 520 and the tripeptide $\mathrm{CAC}$ in $\mathrm{H}_{2} \mathrm{O}: \mathrm{CH}_{3} \mathrm{OH}$ following photoexcitation at $520 \mathrm{~nm}$. Also shown is the reconstructed gas phase fluorescence intensity ( $I_{\text {reconstruct }}=$ $\left(F I_{\text {max }} * I_{S G}\right)+\left(F I_{\text {end }} * I_{D G}\right)$ with $F I_{\max }=1.1$ and $F I_{\text {end }}=$ 0.9 , see text for details) using the ion intensities shown in Figure $\mathrm{S} 1$ for singly and doubly tagged CAC. The dark grey curve is a 10 point adjacent averaged smoothing of the raw data.

and gas phase measurements of a system where self-quenching is expected to be important. For these exploratory experiments, Atto 520 maleimide (A520, Figure S1) was chosen as a prototype chromophore, since this is a commercially available bright fluorescent dye with absorption maximum at $524 \mathrm{~nm}$ and fluorescence maximum at $546 \mathrm{~nm}$ in $\mathrm{H}_{2} \mathrm{O} / \mathrm{CH}_{3} \mathrm{OH}$ (Figure $\mathrm{S} 2$ ). The uncapped tripeptide $\mathrm{H}-\mathrm{CAC}-\mathrm{OH}$ was chosen for tagging as chromophores are forced into close proximity upon tagging of such a small system and hence should undergo selfquenching.

To test this hypothesis, the fluorescence intensity of A520 at $543 \mathrm{~nm}$ following excitation at $520 \mathrm{~nm}$ was monitored as a function of time following mixture in a $\sim 2: 1$ ratio with CAC, all in 1:1 $\mathrm{H}_{2} \mathrm{O}: \mathrm{CH}_{3} \mathrm{OH}$ at $\sim 1 \mu \mathrm{M}$, Figure 1. Two different behaviors may be observed; an initial increase in the fluorescence intensity, with a maximum at around 1.10 followed by a subsequent decrease tending towards 0.9. To further explore how the fluorescence behavior correlates with tagging of CAC with A520, the relative intensity of singly and doubly tagged CAC following ESI of an identical solution were measured as a function of the time (Figure S3). Comparison of the gas and solution phase measurements suggest that the initial rise in the fluorescence can be attributed to formation of singly tagged CAC, implying that tagging of the chromophore increases its fluorescence quantum yield (as indicated by AttoTech in their brochure). A similar change in photophysics occurs in Eosin Y maleimide, where fluorescence is only observed upon the reduction of the maleimide to succinimide by covalent tagging. ${ }^{39}$ The increase in the doubly tagged CAC lags behind singly tagged since formation of the singly tagged peptide is a prerequisite for the doubly tagged species. Using the relative intensity of the single and doubly tagged species in the mass spectra, it is possible to reconstruct the fluorescence intensity using the formula $I_{\text {reconstruct }}=\left(F I_{\max } * I_{S G}\right)+\left(F I_{\text {end }} * I_{D G}\right)$ where $F I_{\max }=1.1$ is the fluorescence intensity of the singly tagged CAC, $F I_{\text {end }}=0.9$, Figure 1 . The reconstructed fluorescence intensity closely matches the observed solution phase measurements, indicating that it is the presence of doubly 


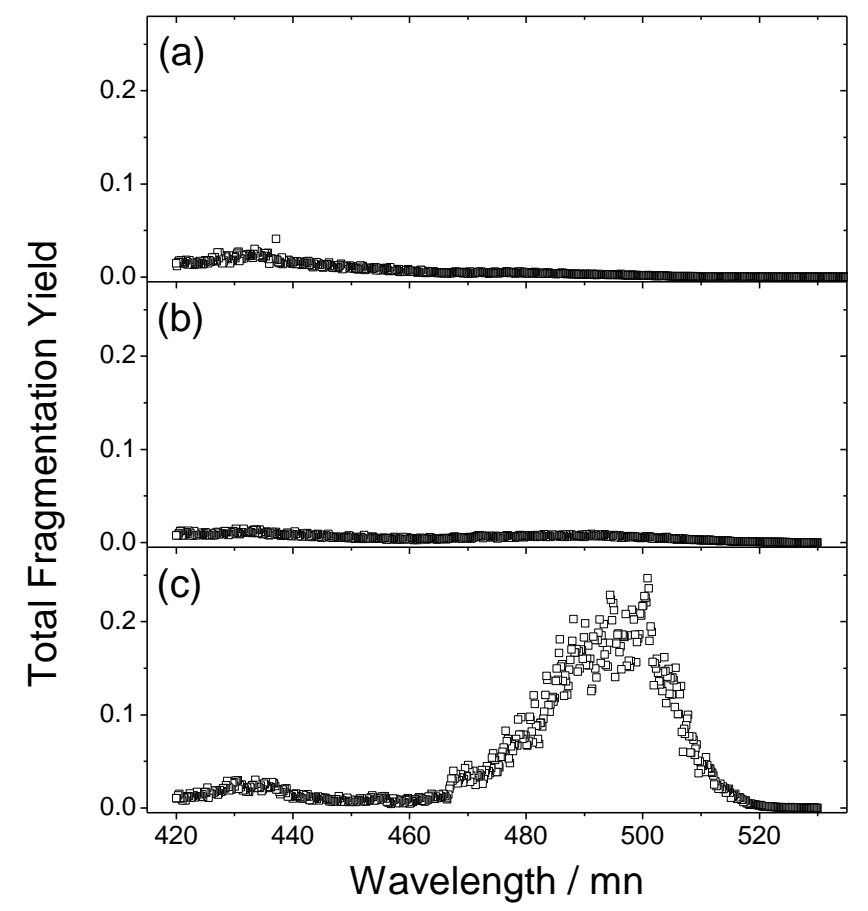

Figure 2. Action-spectra of mass selected (a) $[\mathrm{A} 520]^{+}$, (b) $[\mathrm{A} 520-$ $\mathrm{CAC}^{+}$, and (c) [A520-CAC-A520] ${ }^{2+}$.

tagged CAC inducing dimers of A520 which is responsible for the decrease in fluorescence intensity.

How does this change in relaxation pathway upon dimerization present itself in gas-phase measurements of actionspectra? Figures 2 and S4 show the action spectrum for isolated A520 (a), singly tagged CAC (b) and doubly tagged CAC (c), where the same scale is used for direct comparison. That there is a marked increase in the fragmentation efficiency in the doubly tagged species is evident, whilst where there is a single chromophore very low fragmentation efficiency is observed; the latter being expected for a fluorescent dye where de-excitation occurs predominantly by photon emission. The maximum of fragmentation for the isolated chromophore is found at $435 \mathrm{~nm}$, which significantly blue-shifted with regard to the solution phase value of $520 \mathrm{~nm}$. It has been previously established that photofragmentation of fluorescent rhodamine derivatives occurs via resonant two photon absorption via the $\mathrm{S}_{1}$ state to higher lying singlet states. ${ }^{42}$ This explains the difference in the action spectrum for the isolated chromophore as compared to the solution-phase absorption spectrum. Since two photons are absorbed, the fragmentation efficiency is sensitive to the absorption cross-section not just of $S_{0}$ to $S_{1}$ but from $S_{1}$ to higher lying singlet states (see Figure S5).

The maximum in the action spectrum for doubly tagged CAC is $495 \mathrm{~nm}$ which is similar to the value obtained for a similar dimer of Rhodamine $575 .^{16}$ A previous computational study on dimers of rhodamine B showed that an optically dark state exists at lower energy than the first optically bright transition. $^{34}$ Kasha's rule states that appreciable fluorescence occurs only from the lowest lying excited state of a given multiplicity, and since the $S_{1}$ state in the dimer was calculated to be optically dark, there can be no fluorescence and hence alternative relaxation mechanisms such as internal conversion must occur, leading to photofragmentation.

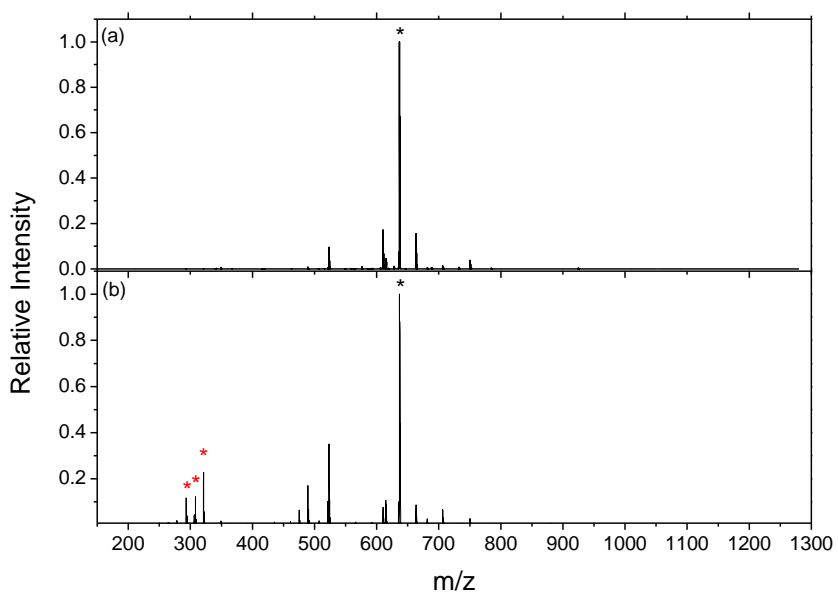

Figure 3. Mass spectra of mass selected [A520-CAC-A520] ${ }^{2+}$ following CID (a) or irradiation with a single laser pulse of 495 $\mathrm{nm}$ (b). The black asterisks denotes the parent cation, and the red asterisks denote the chromophore specific fragmentation associated with the self-quenching.

It is also interesting to note that photo-specific fragmentation is observed, i.e. photo-fragments that are not seen after collision induced dissociation (CID) see Figure 3 (and also Tables S1-S2 and Figure S6 for assignments). This indicates that fragmentation following laser induced dissociation (LID) is really located on the chromophore, which is not observed for collision induced dissociation where the energy is isotropically distributed. In particular the fragments labelled with asterisks on Figure 3 are far more intense following LID at $495 \mathrm{~nm}$, and in a manner analogous to that used in actionFRET they can be used as a reporter that self-quenching has occurred. $^{16}$

In order to probe the influence of the chromophore separation on the self-quenching, the 12-28 fragment of amyloidbeta with capped cysteine residues at both termini $(\mathrm{Ca \beta C})$ was chosen as a prototype system, as the structure has already been characterized in the group. ${ }^{17}$ Previous measurements particularly focused on changes to the structure for $4+, 5+$ and $6+$ doubly tagged cations using action-FRET. This provides an interesting test of the use of self-quenching as a useful probe of chromophore separation, since it is conformationally changes between different compact structures which selfquenching is expected to be sensitive to. Indeed, upon ESI of doubly tagged $\mathrm{CA} \beta \mathrm{C}(12-28)$ charge states from $2+$ to $6+$ are observed (an isobaric species precludes isolation of the 6+ charge state and hence this is not considered further).

Figure 4 shows the action spectra for mass selected doubly tagged $\mathrm{CA} \beta \mathrm{C}(12-28)$ in the $2+$ to $5+$ charge states. There are two important trends that can be noted here; the first is that the intensity of the band at $495 \mathrm{~nm}$ decreases as the charge state increases, and a band at $435 \mathrm{~nm}$ is observed as the charge state is increased. The action spectrum for the $2+$ charge state is essentially identical to that observed in Figure 2(c) and indicates that both chromophores are very close, as expected for a very compact structure. The action spectra for the $4+$ and $5+$ charge states resemble very closely those observed for isolated chromophores shown in Figure 2(a-b). This is consistent with previous measurements which showed a change in secondary 


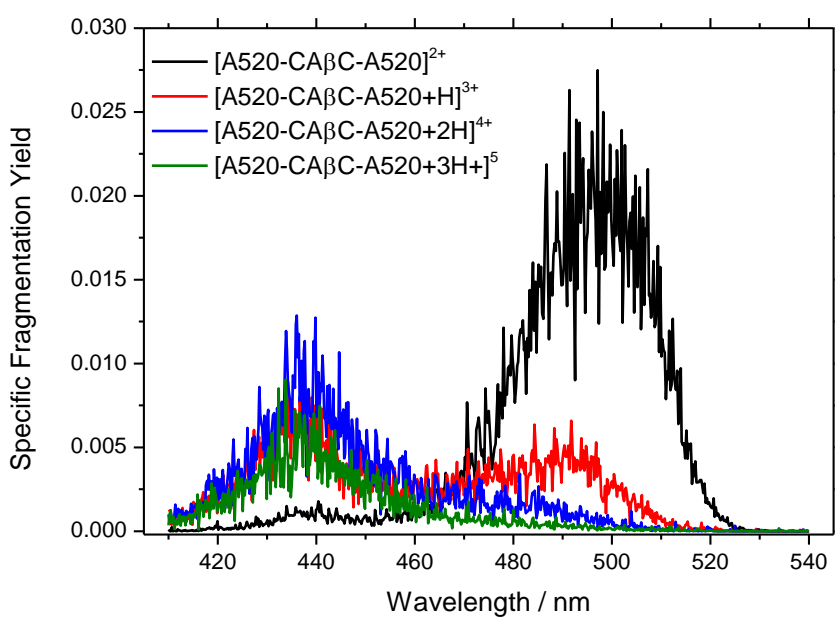

Figure 4. Action spectra of mass selected doubly tagged $\mathrm{CA} \beta \mathrm{C}_{12-}$ ${ }_{28}$ in the $2+$ (black), 3+ (red), 4+ (blue) and 5+ (green) charge states.

structure from random coil to alpha-helical between $3+$ and $4+$ charge states, resulting in a large change in chromophore separation from $\sim 10 \AA$ to $\sim 40 \AA$, making chromophore dimer formation impossible. ${ }^{17}$ The band at $435 \mathrm{~nm}$ can therefore be assigned as an intense resonant two-photon absorption discussed above.

Why is the band at $435 \mathrm{~nm}$ not observed when the system is undergoing self-quenching? In the case of the isolated chromophore it has been previously measured for similar rhodamine derivatives that the $S_{1}$ state has a lifetime of approximately $5 \mathrm{~ns}^{43}$ Solution phase measurements of the fluorescence lifetimes of rhodamine dyes at high concentrations have found lifetimes as low as $1 \mathrm{ps,} \mathrm{indicating} \mathrm{that} \mathrm{the} \mathrm{initially}$ populated bright state is extremely short-lived in dimeric species. ${ }^{33}$ Hence, the suppression of the band at $435 \mathrm{~nm}$ in the $2+$ could be explained by a decrease in the lifetime of the initially excited singlet state, yielding subsequent decrease in the probability for absorption of a second photon. In this case, Figure $2 \mathrm{c}$ suggests that the small size of the tripeptide prevents a perfect stacking of the chromophores since the $435 \mathrm{~nm}$ band is observed here, which is also consistent with the non-zero fluorescence intensity at long time scales seen in Figure 1.

The $3+$ charge state appears to be an intermediate case to the 2+ and 4+ charge states; with both the 495 and $435 \mathrm{~nm}$ bands observed. This is suggestive of there being at least two distinct conformational families present: one in which the chromophores are close enough to interact and undergo selfquenching, and one in which they are too far apart to form an intra-molecular chromophore dimer. In order to confirm this hypothesis, ion mobility profiles were measured for the $3+, 4+$ and $5+$ charge states of doubly tagged $\mathrm{CA} \beta \mathrm{C}(12-28)$ (it was not possible to observe the $2+$ charge state with sufficient intensity to perform ion-mobility on it), Figure 5. The 4+ and $5+$ charge states are clearly dominated by a single conformational family, as previously observed, corresponding to an extended helical conformation. ${ }^{17}$ The $3+$, however, shows at least two conformational families, which is entirely consistent with the conclusions made from the self-quenching experiments. Furthermore, it must be noted that previous FRETefficiency measurements of the $3+$ charge state gave a value of

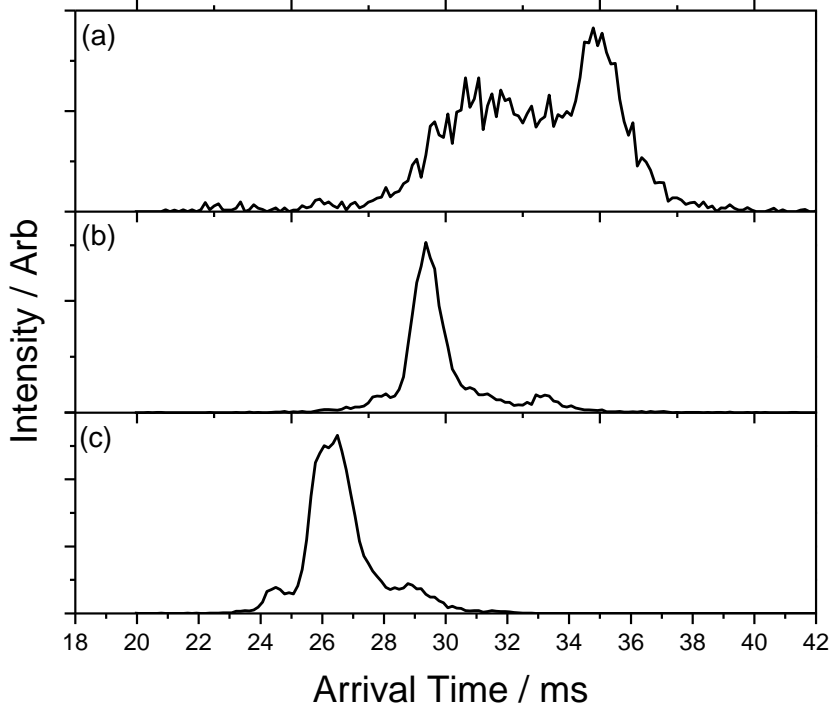

Figure 5. Ion Mobility arrival time distributions for the 3+ (a), 4+ (b) and $5+(\mathrm{c})$ charge states of doubly tagged $\mathrm{Ca} \beta \mathrm{C}(12-28)$.

1 , providing no information other than confirming that chromophores are very close. This therefore indicates that selfquenching is a sensitive probe for structural changes that occur in compact peptide conformations where FRET is not sensitive.

It is also possible to extend fluorescence self-quenching measurements to larger protein systems to probe small-scale changes in conformation here. A double cysteine mutant of ubiquitin which has been used previously for an action-FRET study was used as a prototype system. ${ }^{18}$ In the action-FRET measurements, it was the change from native to unfolder Astate that was being probed. In the case of fluorescence quenching, it will instead be smaller-scale changes in local structure; involving changing of the chromophore conformation relative to the protein structure as charge state is increased.

Figure 6 shows the specific chromophore fragmentation yields for different charge states of ubiquitin produced via ESI of a $\mathrm{H}_{2} \mathrm{O}$ solution with $0.1 \%$ acetic acid by volume. Since the photofragmentation yields are in general much smaller for protein species, a background subtraction was performed as described above in order to give a better estimate of the specific photofragmentation yields. There is a large decrease in the corrected specific photofragmentation yield for the $5+$ and $6+$ charge states, a smaller decrease between $6+$ and $7+$ followed by a constant small value. The large decrease in specific fragmentation yield for the $6+$ compared to the $5+$ can be explained by unfolding of the chromophore linker chain on the same compact protein structure. Such an unfolding was also observed in action-FRET measurements on the same system. ${ }^{18}$ In the case of the $6+$ charge state, the specific fragmentation yield remains above the baseline level seen for higher charge states. This indicates that there is a small population of structures in which the chromophores remain close enough to undergo self-quenching, but the linker chains are predominantly in an unfolded conformation. For the $7+$ and higher charge states, there is no longer the possibility to form an intramolecular chromophore dimer, which is consistent with previous 


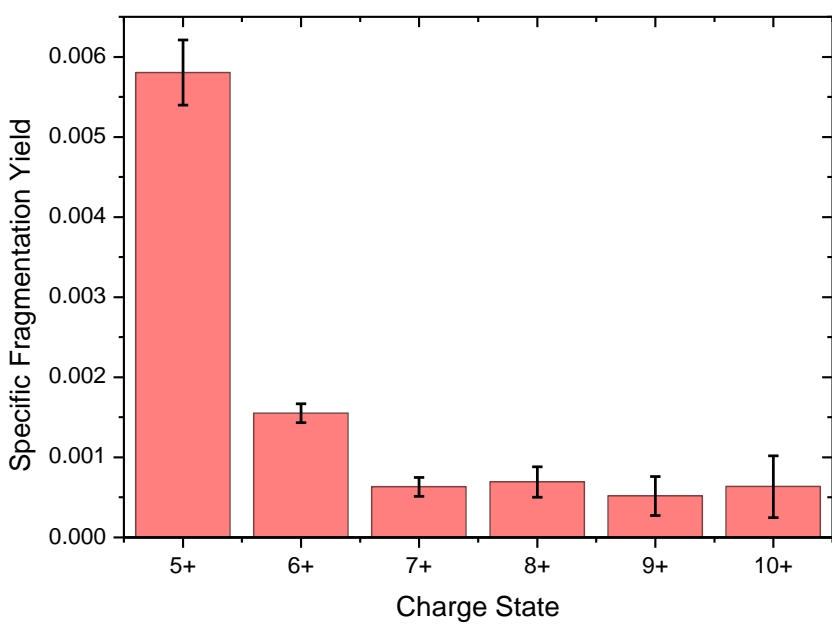

Figure 6. Background corrected specific fragmentation (red bars) associated with self-quenching for doubly tagged ubiquitin cations produced by ESI from $\mathrm{H}_{2} \mathrm{O}$ with $0.1 \%$ acetic acid by volume.

action-FRET measurements and molecular dynamics simulations as well as ion mobility data. ${ }^{18,44-46}$ That the specific photofragmentation yield does not go to zero can be explained by a combination of resonant two-photon absorption of an isolated chromophore leading to fragmentation and an imperfect background subtraction.

\section{Conclusions and Prospects.}

The phenomenon of fluorescence self-quenching in Atto 520 has been studied in both solution and gas phases by fluorescence spectroscopy, mass spectrometry, action spectroscopy and ion mobility mass spectrometry. Solution-phase fluorescence intensity measurements in combination with mass spectrometry measurements of relative intensity of doubly tagged CAC showed that an initial rise in fluorescence intensity can be attributed to singly tagging, a subsequent decrease is associated with double tagging and the formation of chromophore dimers. Comparison of the gas-phase action-spectra of isolated chromophore and singly and doubly tagged CAC showed that a large increase in the fragmentation yield originating from a change in the photophysical properties are observed for doubly tagged species.

It was shown both by examination tagged versions of the 12-28 fragment of amyloid-beta and ubiquitin that selfquenching is indeed a sensitive probe of biomolecule structure. In particular, the action-spectra for different charge states of the tagged amyloid-beta fragment resemble either the test case for an intramolecular chromophore dimer, or the isolated monomer. In the case of the $3+$, the presence of two conformational families - one in which dimer formation is possible and one where it is not - were inferred from the signatures in the action spectrum of both dimeric and isolated chromophores, which was confirmed by observation of a bimodal ion mobility profile. This observation demonstrates nicely the complementarity of action self-quenching and action-FRET, since previous action-FRET measurements of the same system did not show evidence for two conformational families. ${ }^{17}$ In the case of ubiquitin, it was shown that action self-quenching scales to larger systems.

From the aforementioned observations, measurement of the level of action self-quenching can be measured in the follow- ing way. The first would be to examine the ratio of specific fragmentation yield at $495 \mathrm{~nm}$ compared to $435 \mathrm{~nm}$; the higher the ratio, the greater the proportion of the conformations that allow intramolecular dimer formation. Since self-quenching shows an essentially on-off response, it is well suited for studying either association or dissociation of different regions of a protein after performing some activation - binding or removal of a ligand to/from a protein, for example, or probing how individual proteins within a larger protein complex interact with each other.

\section{ASSOCIATED CONTENT}

\section{Supporting Information}

Chromophore structure, solution and gas phase properties of monomeric and dimeric species, and a fragmentation scheme for collision and laser induced dissociation is included in as the supplimentary information (pdf). The Supporting Information is available free of charge on the ACS Publications website.

\section{AUTHOR INFORMATION}

\section{Corresponding Author}

*Phillipe.dugourd@univ-lyon1.fr

\section{Current Address}

* Mass Spectrometry \& Advanced Instrumentation Group, Korea Basic Science Institute (KBSI), Chungbuk, Republic of Korea.

\section{Author Contributions}

The manuscript was written through contributions of all authors.

\section{ACKNOWLEDGMENT}

The research leading to these results has received funding from the European Research Council under the European Union's Seventh Framework Programme (FP7/2007-2013 Grant Agreement No. 320659).

\section{REFERENCES}

(1) Giepmans, B. N. G. Science. 2006, 312, 217-224.

(2) Singh, A. P.; Wohland, T. Current Opinion in Chemical Biology. 2014, pp 29-35.

(3) Jares-Erijman, E. A.; Jovin, T. M. Nat. Biotechnol. 2003, 21, 1387-1395.

(4) Wallrabe, H.; Periasamy, A. Curr. Opin. Biotechnol. 2005, 16, 19-27.

(5) Weiss, S. Science (80-. ). 1999, 283, 1676-1683.

(6) Huang, B.; Wang, W.; Bates, M.; Zhuang, X. Science (80-. ). 2008, 319, 810-813.

(7) Pawlicki, M.; Collins, H. A.; Denning, R. G.; Anderson, H. L. Angew. Chemie Int. Ed. 2009, 48, 3244-3266.

(8) Heck, A. J. R. Nat. Methods 2008, 5, 927-933.

(9) van den Heuvel, R. H.; Heck, A. J. R. Curr. Opin. Chem. Biol. 2004, 8, 519-526.

(10) Wysocki, V. H.; Resing, K. A.; Zhang, Q.; Cheng, G. Methods. 2005, pp 211-222.

(11) Uetrecht, C.; Rose, R. J.; van Duijn, E.; Lorenzen, K.; Heck, A. J. R. Chem. Soc. Rev. 2010, 39, 1633-1655.

(12) Danell, A. S.; Parks, J. H. Int. J. Mass Spectrom. 2003, 229, 3545.

(13) Dashtiev, M.; Azov, V.; Frankevich, V.; Scharfenberg, L.; Zenobi, R. J. Am. Soc. Mass Spectrom. 2005, 16, 1481-1487.

(14) Talbot, F. O.; Rullo, A.; Yao, H.; Jockusch, R. A. J. Am. Chem. Soc. 2010, 132, 16156-16164.

(15) Czar, M. F.; Zosel, F.; König, I.; Nettels, D.; Wunderlich, B.; Schuler, B.; Zarrine-Afsar, A.; Jockusch, R. A. Anal. Chem. 
2015, 87, 7559-7565.

Daly, S.; Poussigue, F.; Simon, A.-L.; MacAleese, L.; Bertorelle, F.; Chirot, F.; Antoine, R.; Dugourd, P. Anal. Chem. 2014, 86, 8798-8804.

(17) Daly, S.; Kulesza, A.; Poussigue, F.; Simon, A.-L.; Choi, C. M.; Knight, G.; Chirot, F.; MacAleese, L.; Antoine, R.; Dugourd, P. Chem. Sci. 2015, 6, 5040-5047.

(18) Daly, S.; Knight, G.; Halim, M. A.; Kulesza, A.; Choi, C. M.; Chirot, F.; MacAleese, L.; Antoine, R.; Dugourd, P. J. Am. Soc. Mass Spectrom. 2016.

(19) Hendricks, N. G.; Lareau, N. M.; Stow, S. M.; McLean, J. A.; Julian, R. R. J. Am. Chem. Soc. 2014, 136, 13363-13370.

(20) Hendricks, N. G.; Julian, R. R. Phys. Chem. Chem. Phys. 2015 , 17, 25822-25827.

(21) Zenobi, R. Anal. Chem. 2015, 87, 7497-7498.

(22) Kulesza, A.; Daly, S.; Choi, C. M.; Simon, A.-L.; Chirot, F.; MacAleese, L.; Antoine, R.; Dugourd, P. Phys. Chem. Chem. Phys. 2016, 18, 9061-9069.

(23) Voronina, L.; Masson, A.; Kamrath, M.; Schubert, F.; Clemmer, D.; Baldauf, C.; Rizzo, T. J. Am. Chem. Soc. 2016, 138, 9224 9233.

(24) Beveridge, R.; Phillips, A. S.; Denbigh, L.; Saleem, H. M.; MacPhee, C. E.; Barran, P. E. Proteomics 2015, 15, 2872-2883.

(25) Breuker, K.; McLafferty, F. W. Proc. Natl. Acad. Sci. U. S. A. 2008, 105, 18145-18152.

(26) Chen, S.-H.; Russell, D. H. J. Am. Soc. Mass Spectrom. 2015 , 26, 1433-1443.

(27) Silveira, J. A.; Fort, K. L.; Kim, D.; Servage, K. A.; Pierson, N. A.; Clemmer, D. E.; Russell, D. H. J. Am. Chem. Soc. 2013, 135, 19147-19153.

(28) Kulesza, A.; Daly, S.; MacAleese, L.; Antoine, R.; Dugourd, P. J. Chem. Phys. 2015, 143, 25101.

(29) Kuiper, J. M.; Pluta, R.; Huibers, W. H. C.; Fusetti, F.; Geertsma, E. R.; Poolman, B. Protein Sci. 2009, 18, 1033-1041.

(30) Dumas, A.; Lercher, L.; Spicer, C. D.; Davis, B. G. Chem. Sci. 2015, 6, 50-69.

(31) Kajihara, D.; Abe, R.; Iijima, I.; Komiyama, C.; Sisido, M.; Hohsaka, T. Nat. Methods 2006, 3, 923-929.

(32) Beljonne, D.; Curutchet, C.; Scholes, G. D.; Silbey, R. J. J. Phys. Chem. B 2009, 113, 6583-6599.

(33) Penzkofer, a; Lu, Y. Chem. Phys. 1986, 103, 399-406 STfluorescence quenching of rhodamine.

(34) Setiawan, D.; Kazaryan, A.; Martoprawiro, M. A.; Filatov, M. Phys. Chem. Chem. Phys. 2010, 12, 11238.

(35) Hernando, J.; Van der Schaaf, M.; Van Dijk, E. M. H. P.; Sauer, M.; García-Parajó, M. F.; Van Hulst, N. F. J. Phys. Chem. A 2003, 107, 43-52.

(36) Wendt, H.; Berger, C.; Baici, a; Thomas, R. M.; Bosshard, H. R. Biochemistry 1995, 34, 4097-4107.

(37) Quinn, S. D.; Dalgarno, P. A.; Cameron, R. T.; Hedley, G. J.; Hacker, C.; Lucocq, J. M.; Baillie, G. S.; Samuel, I. D. W.; Penedo, J. C. Mol. BioSyst. 2014, 10, 34-44.

(38) Zhou, R.; Kunzelmann, S.; Webb, M. R.; Ha, T. Nano Lett. 2011, 11, 5482-5488.

(39) Daly, S.; Kulesza, A.; Knight, G.; Macaleese, L.; Antoine, R.; Dugourd, P. J. Phys. Chem. A 2016, 120, 3484-3490.

(40) Simon, A.-L.; Chirot, F.; Choi, C. M.; Clavier, C.; Barbaire, M.; Maurelli, J.; Dagany, X.; MacAleese, L.; Dugourd, P. Rev. Sci. Instrum. 2015, 86, 94101

(41) Choi, C. M.; Simon, A.-L.; Chirot, F.; Kulesza, A.; Knight, G.; Daly, S.; MacAleese, L.; Antoine, R.; Dugourd, P. J. Phys. Chem. B 2016, 120, 709-714.

(42) Daly, S.; Kulesza, A.; Knight, G.; MacAleese, L.; Antoine, R.; Dugourd, P. J. Phys. Chem. A 2015, 119, 5634-5641.

(43) Nagy, A. M.; Talbot, F. O.; Czar, M. F.; Jockusch, R. A. J. Photochem. Photobiol. A Chem. 2012, 244, 47-53.

(44) Shi, H.; Pierson, N. A.; Valentine, S. J.; Clemmer, D. E. J. Phys. Chem. B 2012, 116, 3344-3352.

(45) Wyttenbach, T.; Bowers, M. T. J. Phys. Chem. B 2011, 115, 12266-12275.

(46) Li, J. W.; Taraszka, J. A.; Counterman, A. E.; Clemmer, D. E. Int. J. Mass Spectrom. 1999, 185, 37-47. 


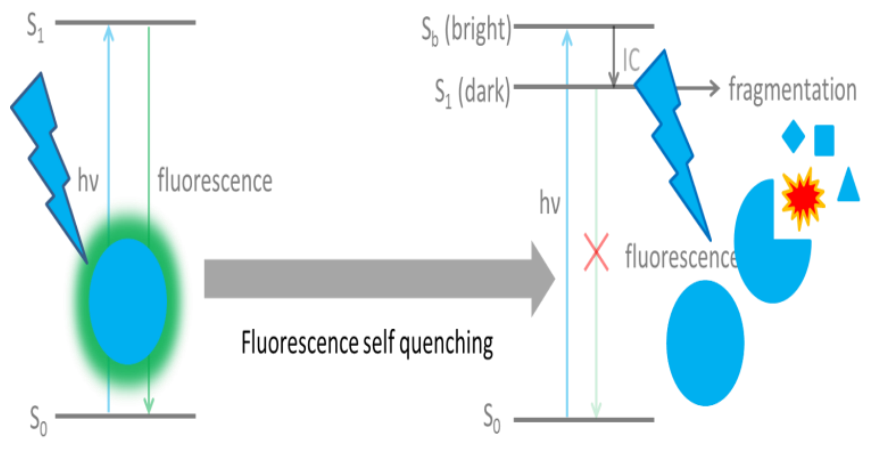

